\title{
Die Zeitschrift sui generis
}

Daniel Hürlimann"

sui generis ist die erste rechtsgebietsübergreifende Open-Access-Zeitschrift der Schweiz. Der nachfolgende Beitrag vermittelt einen Überblick über die Entstehungsgeschichte und die Erfahrungen der seit Zeitschriftengründung vergangenen 5 Jahre.

\section{A. Einleitung}

Die Zeitschrift sui generis (https://sui-generis.ch) ist aus dem Wunsch heraus entstanden, rechtswissenschaftliche Beiträge frei zugänglich zu publizieren. Der Schreibende hatte das Bedürfnis, dass von ihm Geschriebenes gelesen wird und sah nicht ein, warum es im Jahr 2014 noch keine Möglichkeit gab, rechtswissenschaftliche Artikel zu schweizerischem Recht frei zugänglich zu publizieren. Zwar existierten damals in der Schweiz schon zwei rechtswissenschaftliche Open-AccessZeitschriften; diese waren und sind aber auf Nischengebiete spezialisiert. ${ }^{1}$ Deshalb wurde der Entschluss gefasst, eine rechtsgebietsübergreifende juristische Open-Access-Zeitschrift zu gründen.

\section{B. Erste Schritte}

In einem ersten Schritt wurde die Idee einer neuen Zeitschrift, deren Alleinstellungsmerkmal im Open Access bestehen sollte, mit verschiedenen Interessierten diskutiert. Parallel dazu musste eine Webseite gebaut und ein passender Name für die Zeitschrift gefunden werden. In einem zweiten Schritt wurden Redaktionsmitglieder gesucht. Dabei stand das Ziel im Zentrum, von möglichst vielen Rechtsfakultäten schweizerischer Universitäten eine Vertretung im Redaktionsteam zu haben. Sodann mussten Beiträge für die erste Ausgabe akquiriert werden. Diese sollten nach Möglichkeit Themen behandeln, die nicht nur für ein juristisches Publikum von Interesse waren.

Am 31. August 2014 ist die erste Ausgabe der Zeitschrift sui generis erschienen. Das Redaktionsteam bestand aus acht Personen und in der ersten Ausgabe wurden sechs Beiträge publiziert. Die erste Ausgabe war gewissermaßen auch die letzte, weil Beiträge seither nicht mehr gesammelt und zu einem bestimmten Zeitpunkt gemeinsam publiziert, sondern einzeln jeweils im Zeitpunkt der Publikationsreife veröffentlicht werden.

* Dr. Daniel Hürlimann ist Assistenzprofessor für Informationsrecht an der Universität St.Gallen und Herausgeber der Zeitschrift sui generis..

1 Es handelt sich einerseits um die Zeitschrift "Ancilla Iuris - Lagen des Rechts" (www.anci.ch) und andererseits um das "Electronic Journal of Islamic and Middle Eastern Law" (www.ejimel.uzh.ch). 


\section{Technisches}

In den ersten zwei Jahren basierte die Webseite von sui generis auf WordPress, einem weit verbreiteten Content-Management-System, das ursprünglich für Blogs entwickelt wurde. Die grosse Verbreitung der Software bringt konkret spürbare Vor- und Nachteile mit sich. Ein Vorteil ist das grosse Angebot von Plugins. So konnte z.B. ein Plugin gefunden werden, das es erlaubt, anstelle von Fußnoten mit Seitennoten zu arbeiten, d.h. den Fußnotentext nicht am Ende eines Dokuments, sondern rechts neben dem Text (auf der gleichen Höhe wie die entsprechende Fußnotenziffer im Haupttext) anzeigen zu lassen. Aufgrund der Textlaufrichtung (von oben nach unten) und der immer breiter werdenden Bildschirme ist die Idee, Fußnoten neben und nicht unter dem Text anzeigen zu lassen, alles andere als revolutionär. Trotzdem gibt es nur wenige Systeme, welche dies ermöglichen, dazu gehört WordPress. Demgegenüber werden einige Anforderungen, die jede wissenschaftliche Zeitschrift mit sich bringt, von WordPress nicht unterstützt. Ein simples Beispiel sind Co-Autorschaften: Bei WordPress gibt es nur eine Autorin pro Beitrag.

Diese und andere Einschränkungen haben im Laufe der ersten zwei Jahre zum Wunsch geführt, auf ein anderes System umzusteigen. Ein solches war mit Open Journal Systems (OJS) schnell gefunden. OJS ist eine Open-Source-Software für die Publikation von wissenschaftlichen Zeitschriften und wurde vom kanadischen $\mathrm{Pu}$ blic Knowledge Project entwickelt. ${ }^{2}$ Weltweit werden etwa 7000 wissenschaftliche Zeitschriften mit OJS betrieben, ${ }^{3}$ davon 217 in Deutschland, 12 in Österreich und 5 in der Schweiz (Stand 2017). ${ }^{4}$ Seit 2014 wird OJS von der DFG im Rahmen des Projekts "Nachhaltige OJS-Infrastruktur zur elektronischen Publikation wissenschaftlicher Zeitschriften" gefördert. ${ }^{5}$ Die damit finanzierte Plattform OJSde.net "möchte OJS-Akteure im deutschsprachigen Raum vernetzen und Dienstleistungseinrichtungen, Entwickler/innen und Nutzer/innen zusammenbringen". ${ }^{6}$

Für sui generis ist die Umstellung auf OJS im Oktober 2016 erfolgt. Der Zufall wollte es, dass sich zu dieser Zeit an der Universität Zürich das auf OJS basierende Hauptbibliothek Open Publishing Environment (HOPE) im Aufbau befand. ${ }^{7}$ Seit der Umstellung auf OJS verfügen alle Beiträge von sui generis über einen Digital Object Identifier (DOI). Während DOIs in anderen Wissenschaftsdisziplinen längst zum Standard gehören, sind sie in der deutschsprachigen Rechtswissenschaft bis

2 https://pkp.sfu.ca/ojs.

3 https://pkp.sfu.ca/ojs/ojs-usage/ojs-stats.

4 https://pkp.sfu.ca/ojs/ojs-usage/ojs-map.

5 GEPRIS (Geförderte Projekte der DFG), Nachhaltige OJS-Infrastruktur zur elektronischen Publikation wissenschaftlicher Zeitschriften (Projektnummer 245108616, Projektlaufzeit 2014-2017, https://pe rma.cc/HD4R-WSJK) sowie dessen Fortsetzung (Projektnummer 346506625, Förderung seit 2017, https://perma.cc/H8E6-RDH8).

6 Projektbeschreibung auf OJS-de.net (https://perma.cc/ST9S-F7WU).

7 https://www.hope.uzh.ch. 
heute nur vereinzelt anzutreffen. Dies zeigt sich unter anderem daran, dass in juristischen Publikationen darüber sinniert wird, welche digitalen Identifikatoren für juristische Inhalte eingesetzt werden könnten. Eine Untersuchung aus dem Jahr 2018 kommt zum wenig erstaunlichen Ergebnis, "dass die in der Einführung gesetzten Anforderungen mit DOI gut abgedeckt werden könnten". ${ }^{8}$

Seit Februar 2018 werden alle Links in Beiträgen von sui generis über perma.cc geleitet. Bei perma.cc handelt es sich um einen vom Harvard Library Innovation Lab aufgebauten Webarchivierungsdienst. Damit kann ein Abbild einer Webseite im Zeitpunkt ihrer Zitierung erstellt und nachhaltig abrufbar gemacht werden. Dieses Abbild ist auch dann noch zugänglich, wenn die Originalseite nicht mehr existiert. Es handelt sich m.a.W. um die langersehnte Lösung des altbekannten Problems toter Links.

\section{Organisation}

In der Anfangsphase bestand sui generis organisatorisch ausschliesslich aus dem Redaktionsteam mit acht Mitgliedern. Wenige Monate nach dem Erscheinen der Erstausgabe wurde der Verein sui generis gegründet. Dieser wird seither von einem ursprünglich vier- und inzwischen fünfköpfigen und aus Redaktionsmitgliedern bestehenden Vorstand geführt. Im Laufe der Zeit ist das Redaktionsteam auf 16 Mitglieder angewachsen, wobei sämtliche Schweizer Universitäten mit einer Rechtsfakultät in diesem vertreten sind. Die Namen der Mitglieder des Redaktionsteams sind in Anlehnung an das Briefpapier von Anwaltskanzleien auf der Webseite gut sichtbar aufgelistet.

Die Qualitätskontrolle wurde zunächst von Mitgliedern des Redaktionsteams sichergestellt. Im Laufe der Zeit wurden vermehrt auch externe Begutachtungen eingeholt, sodass - auf Anregung eines externen Gutachters - ein Peer Review Board eingerichtet wurde. ${ }^{9}$ Dieses besteht inzwischen aus 48 Mitgliedern, die alle mindestens schon ein Peer Review durchgeführt haben.

Im Jahr 2017 konnte ein studentischer Mitarbeiter in einem 20\%-Pensum angestellt werden. Aufgrund fehlender Finanzierung endete diese Anstellung nach sieben Monaten. Nachdem zusätzliche Finanzierung zugesichert war, konnte im Jahr 2018 wiederum ein Mitarbeiter in einem 20\%-Pensum eingestellt werden. Zusätzlich wird sui generis von einer wissenschaftlichen Assistentin eines Redaktionsmitglieds unterstützt. Im Januar 2019 ist die Schriftleitung von Daniel Hürlimann auf Luca Ranzoni übergegangen.

8 B. Dévaud / F. Kummer, Ein Weg zum eindeutigen digitalen Identifikator juristischer doktrinaler Inhalte, Jusletter IT 22. Februar 2018, Rz. 21.

9 https://sui-generis.ch/peer-review-board. 


\section{E. Finanzierung}

In der Anfangsphase war die Jahresrechnung von sui generis sehr übersichtlich: keine Einnahmen und keine Ausgaben. Da die Zeitschrift ausschliesslich auf Freiwilligenarbeit basierte, konnten in den ersten drei Jahren allerdings nur je acht Beiträge publiziert werden. ${ }^{10}$

Inspiriert von der Idee der Open Library of Humanities ${ }^{11}$ hat der Schreibende im Rahmen eines Referats mit dem Titel "Publishing Open Access from researchers' point of view" beim Konsortium der Schweizer Hochschulbibliotheken ${ }^{12}$ erwähnt, dass geplant sei, Finanzierungsgesuche an Bibliotheken zu richten. Zu seinem Erstaunen fielen die Reaktionen auf diesen Versuchsballon fast ausschliesslich positiv aus. Vertreterinnen und Vertreter von Bibliotheken wollten entsprechende Gesuche erhalten, um diese zum Anlass zu nehmen, grundsätzlich über die Förderung von Open-Access-Zeitschriften zu befinden. Die im Nachgang verschickten Finanzierungsgesuche an zunächst drei Universitätsbibliotheken wurden, wenn auch in sehr unterschiedlichem Umfang, alle gutgeheissen. Mit dem so zusammengekommenen Betrag konnte im Jahr 2017 während sieben Monaten ein studentischer Mitarbeiter zu $20 \%$ beschäftigt werden. Gleichzeitig wurden auch die Akquisitionsbemühungen verstärkt, sodass in diesem Jahr mehr Beiträge als in den ersten drei Jahren zusammen publizierten werden konnten. ${ }^{13}$

Nach einer Vorstellung von sui generis an der Jahrestagung 2017 der Vereinigung der Juristischen Bibliotheken der Schweiz (VJBS) ${ }^{14}$ wurden weitere Finanzierungsgesuche an Universitäts- und Anwaltskanzleibibliotheken verschickt. Während die Universitätsbibliotheken die Gesuche überwiegend gutgeheissen haben, wurden die Gesuche an die Anwaltskanzleibibliotheken entweder gar nicht oder abschlägig beantwortet. Heute wird sui generis von der Hauptbibliothek der Universität Zürich, der Universitätsbibliothek Bern, der Bibliothek der rechtswissenschaftlichen Fakultät der Universität Zürich, der Bibliothek der Universität St.Gallen, der Universitätsbibliothek Basel, der Zentral- und Hochschulbibliothek Luzern, der ZHAW Hochschulbibliothek und der rechtswissenschaftlichen Fakultät der Universität Freiburg (Schweiz) finanziell unterstützt. Die jährlichen Beiträge liegen zwischen CHF 350 und 4000, das Total der finanziellen Förderung durch Bibliotheken betrug im Jahr 2018 CHF 8000. Seit 2018 wird sui generis zusätzlich auch von der schweizerischen Akademie der Geistes- und Sozialwissenschaften finanziell unterstützt.

10 https://sui-generis.ch/issue/archive.

11 https://www.openlibhums.org.

12 https://consortium.ch.

13 Verzeichnis der im Jahr 2017 in sui generis erschienenen Beiträge (https://perma.cc/J2JR-AFP3).

$14 \mathrm{http}: / /$ lawlibraries.ch. 


\section{F. Rezeption}

Auch wenn nicht jeder Klick auf einen Beitrag bedeutet, dass der Klickende ein Mensch ist und dass dieser den Beitrag tatsächlich liest, so kann doch festgehalten werden, dass die in sui generis publizierten Beiträge eine Leserschaft finden. Im Jahr 2018 erzielte der erfolgreichste Beitrag 1900 Klicks, weitere zehn Beiträge haben je zwischen 450 und 1400 Klicks erreicht. Aufgrund der verwendeten CC-Lizenz können die Beiträge auch auf andere Plattformen (z.B. in universitäre Repositorien) hochgeladen oder in anderen Zeitschriften oder Büchern zweitveröffentlicht werden. ${ }^{15}$ sui generis publiziert jedoch keine Beiträge, die bereits andernorts erschienen sind.

sui generis ist - soweit ersichtlich - die einzige juristische Fachzeitschrift der Schweiz, deren Beiträge in Google Scholar aufgenommen werden. Die in sui generis publizierten Beiträge werden auch über einen Newsletter und via Twitter ${ }^{16}$ verbreitet.

In sui generis publizierte Beiträge wurden schon mehrfach in den Medien aufgegriffen. Sowohl die Neue Zürcher Zeitung ${ }^{17}$ als auch der Tagesanzeiger ${ }^{18}$ und das Schweizer Fernsehen ${ }^{19}$ sowie zahlreiche andere Medien haben verschiedentlich Artikel, die in sui generis erschienen sind, direkt verlinkt. Vereinzelt wurden auch Autoren von Beiträgen zu Interviews in Zeitungen, im Radio und im Fernsehen eingeladen. Die in sui generis erscheinenden Beiträge haben auch Eingang in die "Presseschau" von anderen juristischen Zeitschriften gefunden. Schliesslich wird sui generis auch als Beispiel einer erfolgreichen Open-Access-Zeitschrift in den Medien genannt. ${ }^{20}$

\section{G. Meilensteine}

Im April 2015 wurde sui generis in das Directory of Open Access Journals (DOAJ) aufgenommen. Für eine Aufnahme in dieses Verzeichnis müssen insbesondere die

15 So wurde beispielsweise der 2017 publizierte Beitrag von T. Burri, Verfassungslos: Verfassungs- und staatsrechtliche Bedenken anlässlich des Bundesgerichtsentscheides zur Bündner Spracheninitiative, abgedruckt in: D. Thürer/R. Blindenbacher (Hrsg.), Embracing Differences - A Commitment for Minorities and Managing Diversity, Zürich 2018, S. $67 \mathrm{ff}$.

16 https://twitter.com/suigeneris_ch.

17 B. Hürlimann, Justitia wachrütteln, NZZ vom 20. Oktober 2014 (https://perma.cc/X84K-KCQD; Link auf http://sui-generis.ch/8); F. Werro, Konzernverantwortungsinitiative: Mythos Haftung, NZZ vom 18. Dezember 2018 (https://perma.cc/SF6Q-3ZEF; Link auf https://sui-generis.ch/85).

18 P. Loser, Die Fakten zum grössten Wirtschaftsraum aller Zeiten, TagesAnzeiger vom 5. Juni 2015 (https://perma.cc/NNE4-S39T; Link auf http://sui-generis.ch/10).

19 S. Hablützel, Darf die Mehrheit der Minderheit eine Fremdsprache aufzwingen?, SRF Regionaljournal Graubünden vom 10. Oktober 2017 (https://perma.cc/YZ7D-V6JC; Link auf https://sui-generis. $\mathrm{ch} / 40)$.

20 M. Amrein, Open-Access-Zeitschriften: Der Zugang zum Wissen soll endlich frei sein, NZZ am Sonntag vom 17. November 2018. 
folgenden Minimalanforderungen ("basic requirements for inclusion in DOAJ") erfüllt sein: ${ }^{21}$

- Der Volltext aller Artikel muss ab dem Zeitpunkt der Publikation frei zugänglich sein.

- Die Zeitschrift muss über eine eigene Webseite verfügen.

- Jeder Artikel muss über eine eigene URL verfügen.

- Die Zeitschrift muss über eine ISSN (International Standard Serial Number) verfügen.

- Alle Artikel müssen vor der Veröffentlichung ein Qualitätskontrollsystem (Editorial oder Peer Review) durchlaufen und die Art der Überprüfung muss auf der Website angegeben werden.

- Die Website der Zeitschrift muss die Bedingungen für die Nutzung und Weiterverwendung angeben, die Leser und Autoren haben, wenn sie einen Artikel einreichen oder die veröffentlichten Inhalte nutzen.

Ein weiterer Meilenstein war die erstmalige Zitierung eines in sui generis erschienen Beitrags durch das Bundesgericht im Jahr 2016. Diese erfolgte in einem Urteil, mit dem das Bundesgericht über die Beschwerde der Schweizerischen Radio- und Fernsehgesellschaft gegen das Kantonsgericht Graubünden wegen Nichtherausgabe eines Urteils zu befinden hatte. In diesem Urteil wurde einer der ersten in sui generis publizierten Beiträge zitiert. ${ }^{22}$

Im Jahr 2017 konnte aufgrund der Finanzierung durch drei Universitätsbibliotheken erstmals ein studentischer Mitarbeiter angestellt werden. Dies ermöglichte einen deutlichen Anstieg der Anzahl publizierter Beiträge von je 8 in den ersten drei Jahren auf 26 im Jahr 2017.

Seit 2018 arbeiten zwei wissenschaftliche Assistenten zu je ca. 20\% für die Zeitschrift sui generis. Zudem wurde Anfang 2018 vom Editorial Review auf ein doppelblindes Peer Review umgestellt. Das bedeutet, dass die Reviewerinnen die Namen der Autoren nicht kennen und umgekehrt. Trotz Skepsis des Schreibenden, der in dieser Frage überstimmt wurde, waren die Erfahrungen mit dem doppelblinden Peer Review bisher ausschliesslich positiv. Es hat sich gezeigt, dass deutlich mehr Verbesserungsvorschläge gemacht werden, wenn der Name des Autors nicht bekannt ist und die Reviewerin gleichzeitig weiss, dass ihr Name gegenüber dem Autor nicht offengelegt wird. Reviewer haben jedoch auch die Möglichkeit, auf die Anonymität zu verzichten und für direkte Rückfragen zur Verfügung zu stehen. Die Peer Review Guidelines von sui generis sind auf der Webseite abrufbar. ${ }^{23}$

21 Directory of Open Access Journals, Information for Publishers, Ziff. 3: Publishing best practice and basic standards for inclusion (https://perma.cc/WZR8-4HVA).

22 Urteil des Bundesgerichts 1C_123/2016 vom 21. Juni 2016 (https://perma.cc/CL6L-RTQH), E. 3.6.

23 Peer Review Guidelines von sui generis (https://perma.cc/NZ5U-8KRD). 
Ebenfalls seit 2018 ist sui generis stolze Trägerin des DOAJ Seals. Das DOAJ Seal steht für Best Practice im Bereich Open-Access-Publishing. Die Kriterien für das Siegel betreffen die Zugänglichkeit, Offenheit, Auffindbarkeit, Wiederverwendung sowie das Urheberrecht. ${ }^{24}$ Das Siegel wird an Zeitschriften vergeben, welche zusätzlich zu den oben erwähnten allgemeinen DOAJ-Aufnahmekriterien u.a. die folgenden erfüllen: ${ }^{25}$

- Die Zeitschrift muss über eine Archivierungsvereinbarung mit einem externen Anbieter (z.B. CLOCKSS) ${ }^{26}$ verfügen.

- Die in der Zeitschrift publizierten Artikel müssen über einen dauerhaften Identifikator (z.B. DOI $)^{27}$ verfügen.

- Es müssen maschinenlesbare Creative-Commons-Lizenzinformationen in Metadaten auf Artikelebene eingebettet sein.

- Die Zeitschrift muss in mindestens einem Verzeichnis für Weiterverwendungsrechte (“deposit policy registry”), z.B. SHERPA/RoMEO ${ }^{28}$ registriert sein.

- Die Urheberrechte müssen bei den Autorinnen verbleiben.

sui generis erfüllt all diese Bedingungen und wurde deshalb im März 2018 mit dem DOAJ Seal ausgezeichnet. ${ }^{29}$

Im Jahr 2018 wurden 33 Artikel in sui generis publiziert. Aufgrund der erhöhten Qualitätsanforderungen, insbesondere der Einführung des doppelblinden Peer Reviews, wurden mehr Beiträge als in den Vorjahren abgelehnt. Diese erscheinen in aller Regel mit einer gewissen Verzögerung unverändert in anderen juristischen Zeitschriften.

\section{H. Buchreihe}

Im Januar 2018 ist die erste Dissertation in der Schriftenreihe sui generis erschienen. In der Schriftenreihe sui generis werden ausgezeichnete Dissertationen sowie Lehrbücher und Tagungsbände einem breiten Publikum zugänglich gemacht. Die Bücher erscheinen sowohl gedruckt als auch online in den Formaten PDF und ePUB. Die digitale Version ist weltweit für alle Interessierten kostenlos zugänglich. Im Jahr 2018 konnten drei weitere Werke in der Reihe publiziert werden: zwei Dissertationen und das Lehrbuch "Introduction to Swiss Law".

Auch bei den Büchern bleiben die Urheberrechte bei den Autoren und die Werke werden unter einer Creative-Commons-Lizenz veröffentlicht. Die Bücher der Reihe

24 Directory of Open Access Journals, Journal Application Form (https://perma.cc/M6RQ-DCLM).

25 Directory of Open Access Journals, Journal Application Form, Abschnitt "The qualifiers for the DOAJ Seal" (https://perma.cc/M6RQ-DCLM).

26 https://clockss.org.

27 https://www.doi.org.

$28 \mathrm{http} / / / \mathrm{www}$. sherpa.ac.uk/romeo.

29 Erkennbar am Siegel im DOAJ-Eintrag von sui generis (https://perma.cc/7A59-4S54). 
sui generis sind sowohl im Buchhandel als auch kostenlos über die Webseiten der schweizerischen Nationalbibliothek sowie über Google Books, OAPEN und sui-generis.ch/buecher erhältlich. Neu werden in den Büchern sämtliche Gesetzesartikel, Gerichtsurteile und URLs mittels beständiger Permalinks verlinkt. ${ }^{30}$

\section{Ausblick}

Die bestehende personelle und finanzielle Ausstattung von sui generis (Zeitschrift) erlaubt es, maximal 50 Beiträge pro Jahr zu veröffentlichen. Aufgrund der OpenAccess-Strategien von swissuniversities (100\% Open Access bis 2024$)^{31}$ und SNF $(100 \% \text { Open Access bis 2020 })^{32}$ ist davon auszugehen, dass der Bedarf nach Open-Access-Publikationsmöglichkeiten in der Rechtswissenschaft in den kommenden Jahren stark ansteigen wird. Gleichzeitig ist zu beobachten, dass sich die klassischen juristischen Verlage beim Thema Open Access nicht bewegen. Zwar wurden in den letzten Jahren weitere juristische Open-Access-Zeitschriften gegründet. Diese beschränken sich jedoch entweder auf ein bestimmtes Rechtsgebiet (ContraLegem: ${ }^{33}$ Strafrecht) oder auf einen bestimmten Autorenkreis (Cognitio: ${ }^{34}$ studentisches Forum).

Sollte sui generis auch weiterhin die einzige rechtsgebietsübergreifende Open-Access-Zeitschrift der Schweiz bleiben, ist davon auszugehen, dass in Zukunft deutlich mehr Beiträge eingereicht werden. Den zusätzlichen Bedarf an Open-AccessPublikationen kann sui generis nur abdecken, wenn die Zeitschrift mehr Einnahmen generiert, um die Stellenprozente und damit die Publikationskapazität zu erhöhen. Dieser Beitrag soll jedoch nicht mit einem Spendenaufruf enden. sui generis befindet sich in der komfortablen Lage, dass die Finanzierung für den Weiterbetrieb im aktuellen Umfang gewährleistet ist und dass im Bereich des wissenschaftlichen Publizierens Qualität vor Quantität geht. Wenn in Zukunft deutlich mehr Beiträge eingereicht werden, die finanziellen Mittel jedoch nur für die Publikation der 50 besten ausreichen, wird zwangsläufig die Qualität der Beiträge steigen.

30 Das erste Buch, in dem sämtliche Gesetzesartikel, Gerichtsurteile und URLs mittels Permalinks verlinkt sind ist die Dissertation $S$. Hänsenberger, Die zivilrechtliche Haftung für autonome Drohnen unter Einbezug von Zulassungs- und Betriebsvorschriften, Berlin/Bern 2018 (https://perma.cc/584ZLNPP).

31 swissuniversities, Nationale Open-Access-Strategie für die Schweiz (https://perma.cc/Z8X5-SW49).

32 SNF, Ab 2020: SNF-Forschung wird 100\% Open Access (https://perma.cc/5SWA-JK3C).

33 https://www.contralegem.ch.

$34 \mathrm{https} / / / \operatorname{cog}$ nitio-zeitschrift.ch. 